\title{
CD49b, CD87, and CD95 Are Markers for Activated Cancer-Associated Fibroblasts Whereas CD39 Marks Quiescent Normal Fibroblasts in Murine Tumor Models
}

David J. Agorku ${ }^{1,2 *}$, Anne Langhammer ${ }^{1}$, Ute Heider ${ }^{1}$, Stefan Wild ${ }^{1}$, Andreas Bosio ${ }^{1}$ and
Olaf Hardt ${ }^{1}$

${ }^{1}$ Miltenyi Biotec GmbH, Bergisch Gladbach, Germany, ${ }^{2}$ HAN Master Programmes, HAN University of Applied Sciences, Nijmegen, Netherlands

Fibroblasts are thought to be key players in the tumor microenvironment. Means to identify and isolate fibroblasts as well as an understanding of their cancer-specific features are essential to dissect their role in tumor biology. To date, the identification of cancer-associated fibroblasts is widely based on generic markers for activated fibroblasts in combination with their origin in tumor tissue. This study was focused on a deep characterization of the cell surface marker profile of cancer-associated fibroblasts in widely used mouse tumor models and defining aberrant expression profiles by comparing them to their healthy counterparts. We established a generic workflow to isolate healthy and cancer-associated fibroblasts from solid tissues, thereby reducing bias, and background noise introduced by non-target cells. We identified CD87, CD44, CD49b, CD95, and Ly-6C as cancer-associated fibroblast cell surface markers, while CD39 was identified to mark normal fibroblasts from healthy tissues. In addition, we found a functional association of most cancer-related fibroblast markers to proliferation and a systemic upregulation of CD87, and CD49b in tumor-bearing mice, even in non-affected tissues. These novel markers will facilitate the characterization of fibroblasts and shed further light in their functions and implication in cancer progression.

Keywords: cancer-associated fibroblasts, normal fibroblasts, subcutaneous 4T1 mouse tumor model, subcutaneous CT26.WT mouse tumor model, CD39, CD87, CD49b

\section{INTRODUCTION}

Several aspects of tumor growth and progression have been associated with a distinct type of activated fibroblasts, often termed cancer-associated fibroblasts (CAFs) $(1,2)$. It has been reported that CAFs can induce angiogenesis by activating endothelial cells, and pericytes in the tumor microenvironment $(3,4)$, that they can directly affect tumor cells by promoting stemness, and drug resistance (5), and support epithelial-to-mesenchymal transition (EMT) (6). Different regulatory molecules and traits have been identified that are closely associated with the reprogramming and metabolic changes that occur in cancer, many of which have been demonstrated to be affected by fibroblasts (7-10). Via secretion of paracrine factors, CAFs have been shown to regulate the recruitment of inflammatory cells (11). Fibroblasts represent a highly plastic cell type displaying a 
range of activation states. While normal fibroblasts (NFs) are small and thin spindle-shaped cells, cancer-associated fibroblasts are reported to be large, more branched and expressing stress fibers $(1,12)$. Phenotypically, CAFs share many features with activated fibroblasts, e.g., during wound healing, but have mainly been characterized, and detected by intracellular markers and based on patterns of secreted factors. Multiple markers have been used to identify CAFs, including CD44, $\alpha$-smooth muscle actin ( $\alpha \mathrm{SMA})$, fibroblast-activated protein (FAP), fibroblastspecific protein-1 (S100A4), neuron-glial antigen-2 (NG-2), and platelet-derived growth factor $\alpha$, and $\beta$-receptor (PDGFR $\alpha$ and PDGFR $\beta)(5,13,14)$. However, most of these markers can also be detected in NFs, for example upon in vitro propagation based activation. Nevertheless, the role of those markers in CAFs is only poorly understood. Additionally, the expression of CAFmarkers seems to be dependent on the tumor entity which complicates identification and also isolation strategies relying on the expression of defined cell-surface markers. Widely used and accepted markers such as $\alpha$ SMA are intracellular markers and therefore not suitable for identification and isolation of viable cells.

The central role of fibroblasts in the tumor microenvironment and cancer progression has been commonly recognized, however, the identification of markers specific for the cancer-associated phenotype of fibroblasts has not been achieved so far. Therefore, the aim of this study was the identification of CAF-specific cell surface markers in order to facilitate identification and isolation of this cell type from mouse tumor models. As a prerequisite for the identification of cancer-related alterations in fibroblasts, we employed normal fibroblasts isolated from healthy tissues as matched reference population, a highly important control lacking in key publications $(5,15-20)$. This allowed for the identification of novel cell surface markers associated with normal or cancerassociated fibroblasts. Moreover, we were able to show that the expression of those markers correlates to the activation states of fibroblasts.

\section{MATERIALS AND METHODS}

\section{Ethical Guidelines for Animal Research}

All experiments were performed in compliance with European and German guidelines for the care of laboratory animals and were approved by the ethical committee on animal care and use in North Rhine-Westphalia (approval number: 84_02.04.2014.A325).

\section{Mice}

Six to eight week-old BALB/c female mice (Charles River) were used for tumor cell injection and for healthy mammary fat pad and skin. Subcutaneous inoculation was performed by injecting $10^{6}$ cells into the flanks of mice. Tumor growth was monitored by repetitive measurements with calipers and noted as width $\times$ length.

\section{Tissue Dissociation}

Mouse tumor tissue was dissociated into a single cell suspension using the Tumor Dissociation Kit, mouse in combination with the gentleMACS Octo Dissociator with heaters (both Miltenyi Biotec) according to the manufacturer's instructions. Mouse mammary fat pads and skin tissue were dissociated together using Tumor Dissociation Kit, mouse in combination with the gentleMACS Octo Dissociator with heaters (both Miltenyi Biotec) according to manufacturer's instructions. Notably, a custom made protocol was run on the gentleMACS Octo Dissociator with heaters for skin and mammary fat pad from mice. In brief, the tissue was incubated for $1 \mathrm{~h}$ at $37^{\circ} \mathrm{C}$ and under constant rotation at $20 \mathrm{rpm}$, followed by the program 37C_h_TDK_1.

\section{Flow Cytometric Analysis}

For flow cytometric analysis, single cell suspensions were stained with the indicated antibodies (see Materials and Methods and Tables S1, S2) according to the manufacturer's instructions and analyzed using the MACSQuant ${ }^{\mathrm{TM}}$ Analyzer (Miltenyi Biotec). Cells were stained at a density of $0.5-2 \times 10^{5}$ cells per sample in $50 \mu \mathrm{L}$ volume of PBS pH 7.2, 2 mM EDTA, and 0.5\% BSA (PEB) buffer at $2-8^{\circ} \mathrm{C}$ for $10 \mathrm{~min}$, followed by a washing step with $\mathrm{PEB}$. Cell viability was assessed by Propidium Iodide (PI, $1 \mu \mathrm{g} / \mathrm{mL}$, Miltenyi Biotec) prior to flow cytometric analysis. Analysis was performed using the MACSQuant Analyzer and data analysis using the MACSQuatify software (Miltenyi Biotec).

\section{Isolation of CD90.2 Expressing Fibroblasts}

Normal or cancer-associated fibroblasts were isolated by magnetic activated cell sorting (MACS) based on CD90.2 expression. After dissociation fibroblasts were first pre-enriched and then isolated using the Tumor-associated Fibroblast Isolation Kit, mouse (Miltenyi Biotec) with LD- and MScolumns (Miltenyi Biotec), respectively. The isolation procedure was performed according to the manufacturer's instructions. Purity and yield were assessed by flow cytometric analysis using fluorochrome-conjugated CD90.2 antibodies, CD45 antibodies, and Labeling Check Reagent (all Miltenyi Biotec).

\section{Cell Culture}

The mouse breast cancer cell line 4T1 and the colon carcinoma cell line CT26.WT were maintained in Dulbecco's modified Eagle's medium (DMEM, Biowest) supplemented with $10 \%$ fetal calf serum (FCS; Biochrom), $10 \mathrm{mM}$ HEPES (Lonza), $2 \mathrm{mM} \mathrm{L-}$ glutamine (Lonza), and penicillin/streptomycin (Lonza). Cells were maintained at $37^{\circ} \mathrm{C}$ in a humidified $7.5 \% \mathrm{CO}_{2}$ atmosphere.

CD90.2 expressing stromal cells were isolated by MACS from $\mathrm{BALB} / \mathrm{c}$ mouse mammary fat pad and skin or $4 \mathrm{~T} 1$ or CT26.WT tumors generated by subcutaneous injection into BALB/c mice (Charles River). Isolated primary cells were plated on Matrigel (Corning) coated tissue culture ware and grown in DMEM supplemented with $8 \%$ FCS, $10 \mathrm{mM}$ HEPES, $2 \mathrm{mM}$ L-glutamine, $\mathrm{P} / \mathrm{S}$, and $1 \mathrm{x}$ non-essential amino acids (NEAA, Lonza) at $37^{\circ} \mathrm{C}$ in a humidified $7.5 \% \mathrm{CO}_{2}$ atmosphere.

\section{Flow Cytometry-Based Proliferation Analysis}

To determine whether any of the identified surface markers are associated with proliferation, isolated primary cells were stained 
using CellTrace Violet (Life Technologies) according to the manufacturer's instructions, followed by cultivation as previously described. Briefly, cells were washed with PBS after isolation, incubated with $5 \mu \mathrm{M}$ CellTrace Violet at room temperature for $20 \mathrm{~min}$. Next culture medium as described above was added, cells were incubated for additional $5 \mathrm{~min}$ at room temperature, centrifuged at $300 \times \mathrm{g}$ for $5 \mathrm{~min}$ and then resuspended in culture medium. An aliquot of the cells was analyzed using the MACSQuant Analyzer and the rest of the cells was cultivated as described above.

\section{Statistical Analysis}

All statistics and final plots were obtained using GraphPad Prism 6 software and all statistically evaluated experiments were performed in three replicates from at least two independent experiments. For comparison of three different groups (e.g., NFs, 4T1 CAFs, and CT26.WT CAFs), two-way ANOVA was used.

\section{RESULTS}

\section{Generic Isolation of Fibroblast Populations From Solid Tissues}

The aim of the study was the characterization of CAFs from solid mouse tumors, using NFs as matched control populations. In order to identify fibroblast populations in healthy tissues and tumors, we used antibodies recognizing the generic fibroblast marker CD90.2 (Thy-1.2). Thy-1 proteins are widely known markers for fibroblasts from different species and tissues (21-23). We established a method for CD90.2 (Thy-1.2) based isolation of fibroblasts which can be employed for the isolation of both, normal, and cancer-associated fibroblasts. Cells were isolated from dissociated solid tissues by immunomagnetic sorting. As subpopulations of murine leukocytes also express CD90.2 and the starting frequencies of fibroblasts can be very low, in particular in tumor tissues, a two step-procedure was employed to consistently achieve high purity target cells. Non-target cells such as leukocytes and erythrocytes were depleted, followed by a CD90.2-postive selection (Figures 1A-C). Isolated fibroblasts showed a typical morphology in vitro: NFs had a small and compact shape, while CAFs were spindle-shaped with an overall elongated appearance (Figures 1D-G). Of note, NFs grown out of heterogenous bulk cell suspensions showed a CAF-like morphology (Figure 1E), suggesting an activated state and clearly demonstrating the advantage of working with isolated cells as those were not activated in vitro, thus more closely resembling the in vivo state of the cells. In addition, by isolating the target cells a reduction of a bias in downstream analyses by contaminating cell populations is achieved.

\section{Identification of Differentially Expressed Cell Surface Markers Among NFs and CAFs}

Fibroblasts from subcutaneously grown 4T1 and CT26.WT tumors were compared to normal BALB/c mouse skin and mammary fat pad fibroblasts by flow cytometry-based marker screening. The screening was performed using $233 \mathrm{PE}$ conjugated monoclonal or REAfinity antibodies (Table S1). In order to identify fibroblasts within the bulk cells, the samples were co-stained with CD90.2 as generic fibroblast marker as well as CD45 and anti-Ter119 as markers to exclude CD90.2 positive non-fibroblasts (i.e., leukocytes and erythrocytes, respectively). Figures $2 A-D$ shows the gating strategy used for all tissues analyzed in the initial screening, as well as the readout of the analysis with the expression level of each marker (Figures 2E-M). The initial screenings revealed 87 markers expressed by $4 \mathrm{~T} 1$-derived fibroblasts, 87 markers expressed by CT26.WT-derived fibroblasts, and 64 markers expressed on normal fibroblast derived from skin and mammary fat pad of healthy mice. By comparing the markers found to be expressed on the distinct fibroblast populations of the respective tissues with the expression patterns of the leukocyte/erythrocyte and the triple-negative population (CD90.2neg CD45neg Ter119neg), fibroblast-specific markers of the respective tissues were identified (Figures 2E-M). 4T1 tumor-derived fibroblasts showed 16 (out of 87) fibroblastspecific markers, CT26.WT tumor-derived fibroblasts exclusively expressed 9 (out of 87) surface markers, and normal fibroblasts from skin and mammary fat pad showed expression of 9 (out of 64) specific markers. To identify markers differentially expressed among normal and tumor-derived fibroblasts, the markers found to be expressed in the CD90.2pos CD45neg Ter119neg fibroblast populations of the different parental tissues were compared (see Supplementary Figure 1). Among those markers, 12 surface molecules were exclusively found on NFs, while 27 markers were only found on both tumor-derived fibroblast populations. Next to the CAF-related markers, 9 markers specific for the $4 \mathrm{~T} 1$ tumorderived fibroblast population and 12 markers specific for the CT26.WT tumor-derived fibroblast population were identified. Based on these results, a smaller panel of 62 markers was further analyzed and validated (Table S2).

\section{Validation of Candidate Markers on Isolated Fibroblasts}

To validate the most important markers as determined in the initial screening, fibroblasts derived from healthy BALB/c mouse skin, healthy BALB/c mammary fat pad, 4T1, and CT26.WT derived tumors were isolated using CD90.2 immunomagnetic sorting followed by flow cytometry based analysis. The expression of 29 markers could be validated on isolated fibroblasts, while no expression of the remaining 33 markers could be detected after isolation. Hierarchical clustering of the validation data (see Supplementary Figure 2) suggested a close relation of the analyzed CAF populations, while NFs clustered more distinctly. The molecules EphA2, GARP, MHC class II, Rae-1a/b/g, CD80, CD87, CD106, CD123, and CD150 were found to be only expressed on 4T1- and CT26.WT-derived fibroblasts. Yet, only CD87, CD106, and Rae-1a/b/g marked a clear subpopulation ( $>5 \%$ of fibroblasts) in both tumor types, suggesting a cancer-associated regulation rather than a tumor type-related effect. CD39 was clearly overrepresented on normal fibroblasts isolated from "healthy" tissues. In addition 


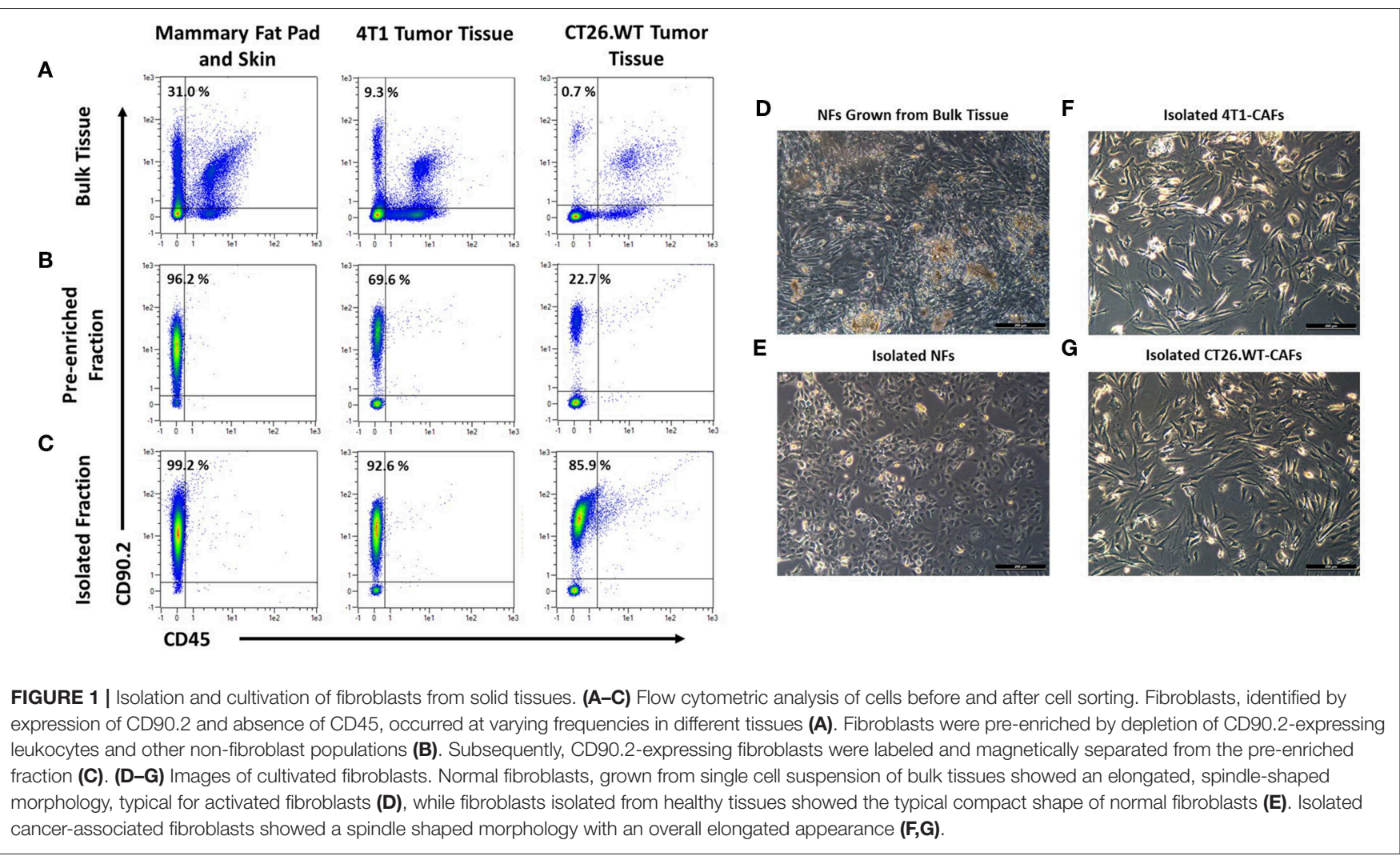

CD95, CD140a, and CD140b were found on a minor fraction of NFs but overrepresented on CAFs. Taken together, CD39 was identified as a potential marker for NFs while the markers CD87, CD106 and Rae-1a/b/g were found to be potential CAF-specific markers. However, CD106, and Rae-1a/b/g were only expressed on a small fraction of CAFs, reducing their value as general CAF markers. Additionally, CD95 showed a marked difference in frequency among normal and cancer-associated fibroblasts. Surprisingly, Ly-6C was found to be expressed on a substantial fraction of all fibroblast populations, although Ly-6C has recently been introduced and discussed as specific marker for a CAF subpopulation termed inflammatory CAFs (iCAFs).

\section{Cultured CAFs Resemble an ex vivo Phenotype While Cultivation of NFs Induces Expression of CAF-Associated Markers}

Comparison of CAFs and NFs revealed a differential expression of CD39 and CD87 ex vivo, next to additional CAF-related cell surface molecules. As many cell-based assays require a period of in vitro propagation, we next asked whether the CAF- and NF-related expression pattern of the most important candidate markers was preserved upon in vitro propagation. Normal fibroblasts were isolated from $\mathrm{BALB} / \mathrm{c}$ mouse skin and fat pad, while 4T1-, and CT26.WT-derived CAFs were isolated from the respective tumor tissue. Cells were analyzed by flow cytometry ex vivo after immunomagnetic cell sorting. Isolated fibroblasts were plated on Matrigel-coated dishes at a density of $1 \times 10^{5} \mathrm{cell} / \mathrm{cm}^{2}$. Cells were grown to a confluency of $\sim 80-90 \%$, usually reached at day 5-6, detached and analyzed again using flow cytometry. Comparison of the acutely isolated ex vivo and in vitro expression profiles of NFs showed that neither the characteristic expression of CD39 nor of Sca-1 was altered in vitro. Notably, a significant increase of NFs expressing CD87 (yet to a comparably low overall level of 20\%), CD95, Ly-6C, and CD44 was observed in vitro (Figure 3A). These data suggested a change of marker expression based on the switch of the functional state upon cultivation toward a more cancer-associated phenotype. In contrast, expression profiles of CAFs were widely preserved in vitro (Figures 3B,C). Only CD44 was significantly reduced in 4T1-derived CAFs after in vitro propagation (Figure 3C), and also decreased in CT26.WTderived CAFs (Figure 3B), indicating a trend for CD44 to decrease in vitro. For the other cell surface markers analyzed no significant induction or loss of surface marker expression was observed.

\section{Increased Expression of CD87, Sca-1, and Ly-6C in NFs Correlates With Their Proliferative Activity}

Upon analysis of fibroblasts in vitro, we found that cultured CAFs resembled the expression of candidates identified in the screening approach, while NFs showed a significantly increased expression of the CAF-associated markers CD44, CD87, CD95, and Ly-6C. As CAFs are described as a distinct 


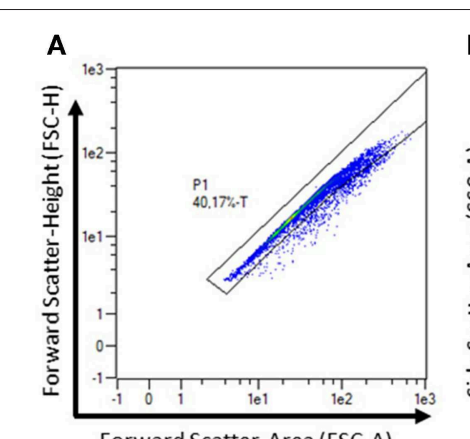

Forward Scatter-Area (FSC-A)

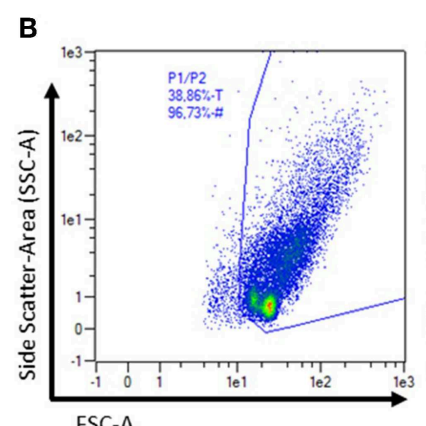

FSC-A c

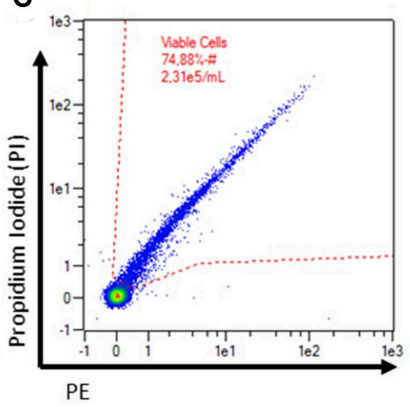

D

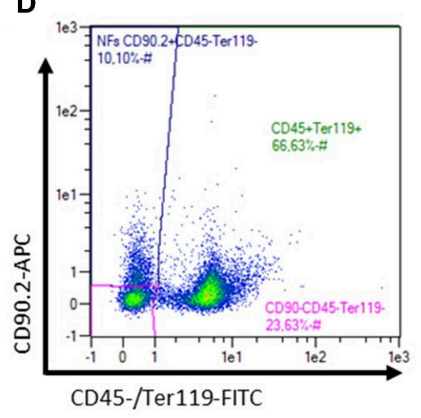

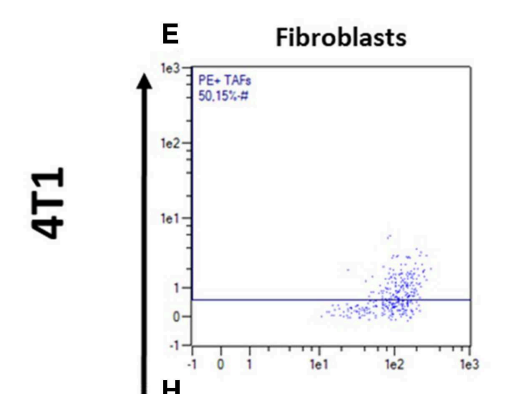

F CD45- CD90.2- Ter119-

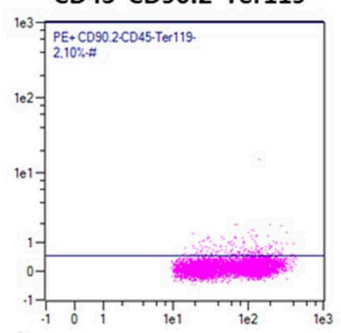

I

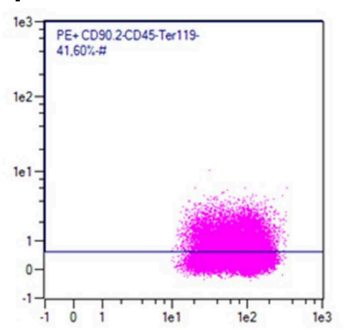

L

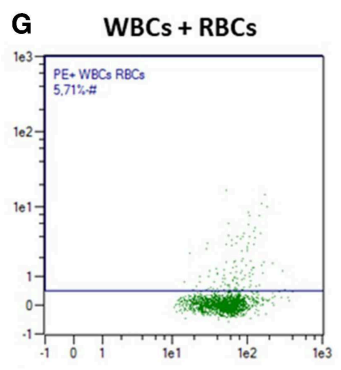

J

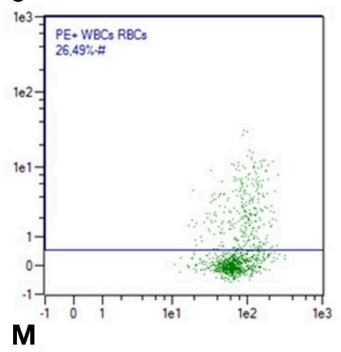

M

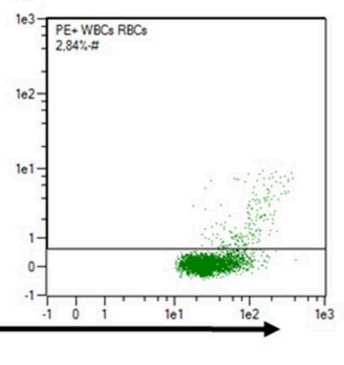

FIGURE 2 | Flow cytometric analysis of fibroblasts from solid tissues. (A-M) Representative gating strategy and read-out for analysis of cell surface marker expression. First, cells are gated in a FSC-A vs. FSC-H plot to exclude doublets (A), followed by exclusion of debris in a FSC-A vs. SSC-A plot (B), and dead cell exclusion on a PE-A vs. PI-A plot (C). Fibroblasts, leukocytes (WBCs), erythrocytes (RBCs), and triple-negative cells were then gated in a plot of FITC-A (CD45 and Ter119) vs. APC-A (CD90.2; D) for independent analysis of the different populations and mean fluorescence intensity levels (E-M). Representative expression patterns of PDGFR $\beta$ (CD140b) are shown for 4T1 and CT26.WT tumor cells as well as for bulk skin and mammary fat pad cells from BALB/c mice.

type of activated fibroblasts and activated fibroblasts proliferate (e.g., during wound healing or inflammation processes), we hypothesized that those markers might be functionally linked to proliferation. To test this, we performed in vitro generational tracing assays. Normal fibroblasts were isolated from healthy $\mathrm{BALB} / \mathrm{c}$ mice by immunomagnetic sorting, stained with a cell trace dye and plated at a density of $1 \times 10^{5}$ cells $/ \mathrm{cm}^{2}$. The cells were grown to a confluency of $\sim 80-90 \%$ (at day 5 after seeding), before they were harvested and analyzed for the expression of CD39, CD44, CD49b, CD87, CD90.2, CD95, Ly$6 \mathrm{C}$, and Sca-1, as well as for their amount of cell divisions by assessing the fluorescence intensity of the cell trace dye. Figures $\mathbf{4 A - E}$ shows the gating for proliferation analysis. The read out is shown in Figures $4 \mathrm{E}-\mathrm{M}$ : populations of fibroblasts that had undergone different numbers of cell divisions were gated based on the fluorescence intensity of the cell trace dye (Figure 4E) and the marker expression was assessed for those populations (Figures $4 \mathbf{F}-\mathbf{M}$ ). Based on the amount of 

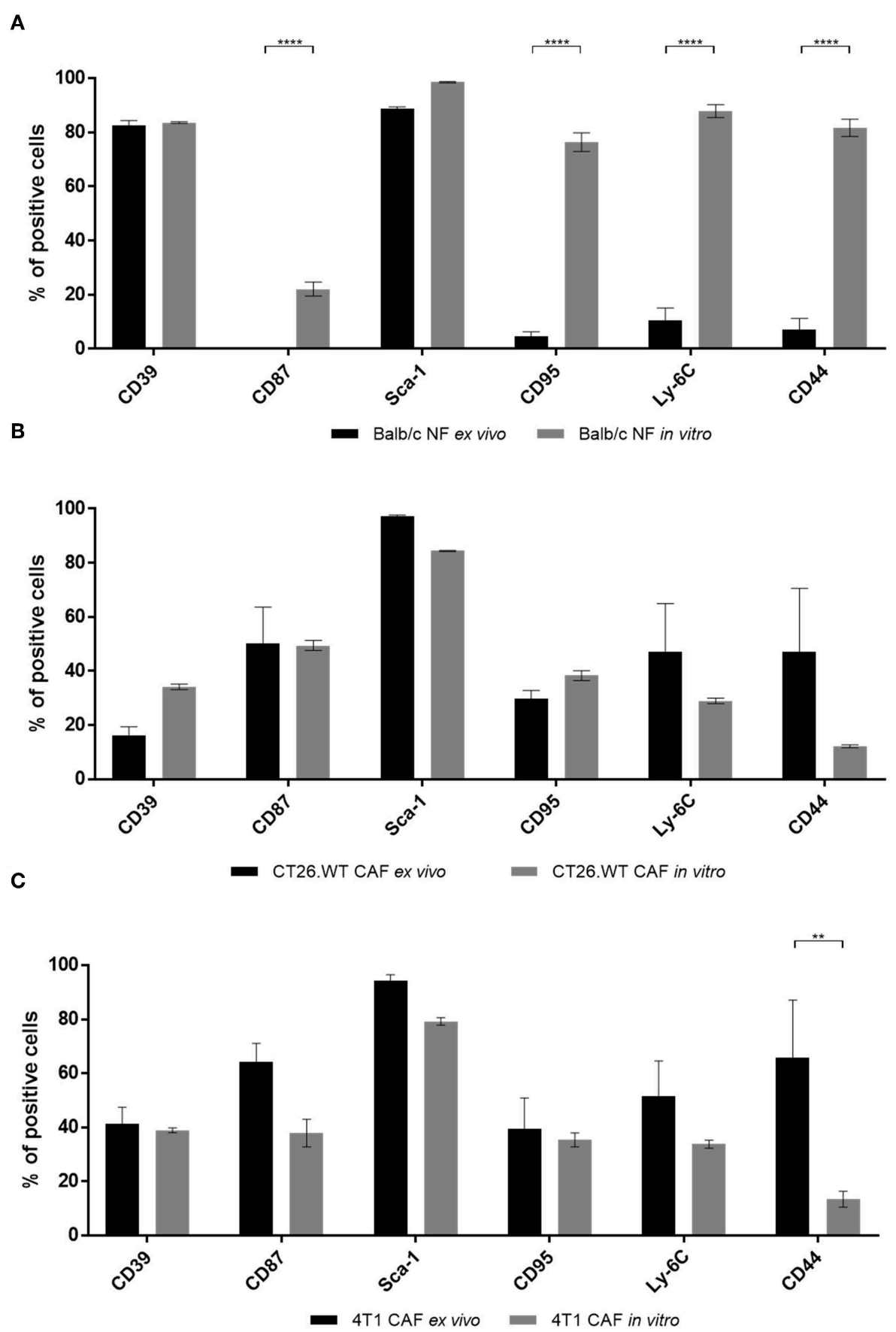

FIGURE 3 | Comparison of ex vivo vs. in vitro phenotype of fibroblasts. (A-C) Phenotyping of ex vivo and in vitro fibroblast populations. NFs (A), CT26.WT- (B), and 4T1-CAFs (C) were analyzed for their expression of CD39, CD87, Sca-1, CD95, Ly-6C, and CD44 before and after in vitro culture. Isolated fibroblasts were seeded at a density of $10^{5} \mathrm{cells} / \mathrm{cm}^{2}$. Analysis was performed when the cells reached a confluency of 80-90\%, in these experiments at day 5 of cultivation. The percentage of cells expressing the respective marker is depicted. Data are reported as median and SEMs for three replicates from at least two independent experiments and statistical analysis was performed using the two-way ANOVA $\left(^{\star \star} P<0.005 ;{ }^{* \star \star *} P<0.0001\right)$.

cells expressing the respective marker (Figure $4 \mathbf{N}$ ) as well as the MFI among the respective subpopulations, a conclusion could be drawn whether the expression pattern correlated with proliferation.
The marker CD49b showed only low expression at comparable levels throughout the different populations and independent of the number of cell divisions. For the markers CD87, CD95, and CD44 an overall weak expression was detected 
as well (Figure 40). However, the percentage of cells expressing those markers was markedly higher as compared to CD49b. Expression of CD90.2 was found among a comparable fraction of cells in all populations, independent of the number of cell divisions. Yet, the level of CD90.2 expression was significantly weaker in the fibroblast population of resting cells and cells that had undergone most cell doublings. The MFI and percentage of Sca-1-expressing cells detected within the population of resting fibroblasts was significantly lower than in cells that had undergone up to two cell divisions. However, cells that had undergone more than two cell divisions showed the same expression levels as resting fibroblasts. In comparison to resting cells, the number of cells expressing CD39 was found slightly increased in the proliferating populations, but was significantly lower in cells that had undergone most cell divisions. Additionally, the MFI of CD39 was significantly lower in cells with increasing numbers of cell divisions. The CAF-associated marker CD87 was found expressed on a subpopulation of NFs propagated in vitro. In contrast to the expression levels detected in CAFs ex vivo and in vitro, the MFI was found to be markedly lower in the NF subpopulations (MFI 0.4-0.9). The frequency of CD87-positive cells was significantly lower in resting fibroblasts compared to the cells that had undergone the first cell division. Interestingly, the population that had undergone most cell divisions showed even significantly lower fractions of CD87-expressing cells. In vitro propagated normal fibroblasts additionally showed differential expression of Ly-6C among the populations with different proliferation behavior. Upon proliferation, the fraction of Ly-6C-positive cells was found significantly increased, while the level of expression was not altered. The cell surface markers CD95 and CD44 showed similar expression profiles: resting cells showed significantly lower frequencies of cells expressing the respective marker, while the MFI showed no significant changes. Notably, cells that had undergone most cell divisions showed a significantly lower frequency of CD95-positive cells compared to resting cells. However, the overall expression level of CD95 and CD44 was very low (MFI 0.5-1 for CD95; MFI 1.0-1.6 for CD44).

Taken together, the expression levels of CD44, CD87, CD95, Sca-1, and Ly-6C positively correlated with activation, indicated by (initial) cell proliferation. However, the levels of CD44, CD87, and CD95 detected in NFs was still very low as compared to CAFs. Moreover, CD87 expression decreased after the second division, indicating that its expression is rather associated with activation than with proliferation per se. The expression level of CD90.2 was also found to be positively correlated with increased cell division, while CD39 showed a decrease in expression upon proliferation. The latter suggests an association of this marker with quiescence in NFs.

\section{"Healthy" NFs Are Marked by CD39 but Express CAF-Markers CD87 and CD49b in Tumor-Bearing Mice}

After the analysis of NFs in vitro suggested a correlation of the CAF-associated markers with proliferation behavior, we sought to analyze NFs from tumor-bearing mice for their specific expression profiles. To the best of our knowledge, such data were completely missing to date. As tumors are thought to shape pre-metastatic niches in preparation of metastasizing (24, 25), we hypothesized that the expression of CAF-associated markers might be induced by such a mechanism leading to increased expression on NFs isolated from tumor-bearing mice. This would demonstrate an additional cancer-specific regulation mechanism of those markers. To test this hypotheses, skin and mammary fat pad of the flank without injected tumor cells was dissected and dissociated into a single cell suspension (Figure 5A). Fibroblasts were isolated from this "healthy tissue" and compared to CAFs isolated from the corresponding tumor as well as to normal fibroblasts isolated from healthy mice. Interestingly, the comparison revealed a mixed phenotype for NFs isolated from tumor-bearing mice (Figures 5B,C). In CT26.WT-derived tumor bearing mice, CD87 and CD49b were significantly overexpressed on fibroblasts (CT26.WT-NFs and CT26.WT-CAFs) in comparison to NFs from healthy mice (Figure 5B). In contrast, CD39 was significantly overexpressed only by CT26.WT-NFs and at the same level as found in NFs from healthy mice, while CD44 was significantly underrepresented in NFs from tumor mice and healthy mice in comparison to CAFs. Notably, CD95 expression in CT26.WTNFs showed a trend toward the levels of "healthy" NFs and being underrepresented in comparison to CT26.WT-CAFs. Normal fibroblasts from $4 \mathrm{~T} 1$ tumor-bearing mice showed the same expression patterns as CT26.WT-NFs for the markers CD39, CD87, CD44, and CD49b. In addition, CD95 and Ly-6C were significantly underrepresented in 4T1-NFs in comparison to 4T1-CAFs (Figure 5C).

Taken together, we identified CD39 as novel cell surface marker for normal fibroblasts in healthy as well as tumor-bearing mice. The expression of CD39 in NFs seemed to be negatively correlated with proliferation. In addition, our data suggested a cancer-associated expression of CD44, CD49b, CD87, CD95, and Ly-6C. Yet, they seemed to correlate with proliferation and accordingly were also found upregulated in proliferating NFs. Of note, CD49b and CD87 seemed to be upregulated in tumorbearing mice in response to a tumor-associated systemic effect.

\section{DISCUSSION}

Many studies on CAFs rely on widely accepted markers for their identification but lack NFs as highly important control when describing and characterizing cancer-associated alterations $(5,15-20)$. Here, we demonstrated the identification of CAFas well as NF-associated cell surface markers by systematically comparing these two subtypes of fibroblasts.

So far, mainly intracellular markers, such as $\alpha \operatorname{SMA}(2,26$, 27), have been identified as suitable markers for distinguishing NFs and CAFs. Moreover, cell surface markers such as the PDGF receptors PDGFR $\alpha$ and PDGFR $\beta$ (27-29) are widely used to identify or isolate CAFs. Using a screening panel of 233 antibodies, the surface marker expression profiles of fibroblasts from healthy skin, healthy mammary fat pad, as well as subcutaneously induced 4T1- and CT26.WT-tumors were 
Agorku et al.

Novel Markers for Cancer-Associated Fibroblasts

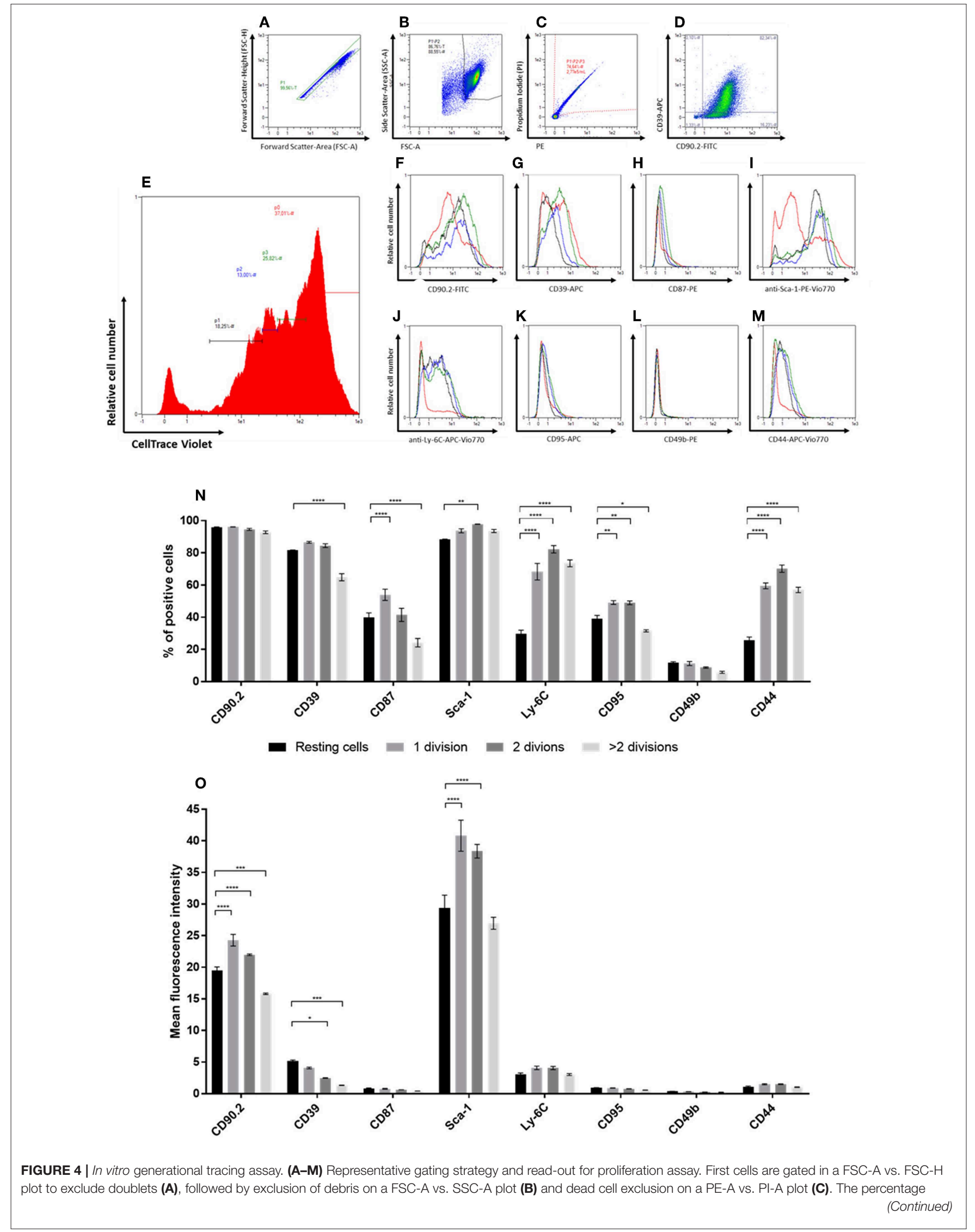

Frontiers in Oncology | www.frontiersin.org

8

August 2019 | Volume 9 | Article 716 
FIGURE 4 | of fibroblasts and the "NF phenotype" is confirmed in a FITC-A (CD90.2) vs. APC-A (CD39; D) plot. For proliferation analysis, populations with different numbers of cell divisions, represented by different peaks, are gated in a histogram indicating the CellTrace Violet fluorescence intensities (E). The peak of cells with the highest CellTrace Violet fluorescence represented cells that did not divide, accordingly referred to as "resting" cells (red). The peaks with gradually lower CellTrace Violet fluorescence represented cell populations with increasing numbers of cell divisions, referred to as "1 division (green)," "2 divisions (blue)," and " $>2$ divisions (black)," respectively. Histograms of the populations with different numbers of cell divisions in vitro indicate the levels of expression for the different markers (CD90.2, CD39, CD87, Sca-1, Ly-6C, CD95, CD49b, and CD44; F-M). (N,0) The expression levels of the different markers within populations after distinct numbers of cell divisions are compared based on the percentage of cells expressing the respective marker ( $\mathbf{N}$ ) and expression level depicted as mean fluorescence intensities (MFIs) of the respective markers (0). Proliferation analysis has been performed in triplicates in two independent experiments. Data are reported as mean and SEMs for three replicates and statistical analysis was performed using the two-way ANOVA ( $\left.{ }^{\star} P<0.05 ;{ }^{\star \star} P<0.005 ;{ }^{{ }^{\star \star}} P<0.0005 ;{ }^{\star \star \star \star} P<0.0001\right)$.

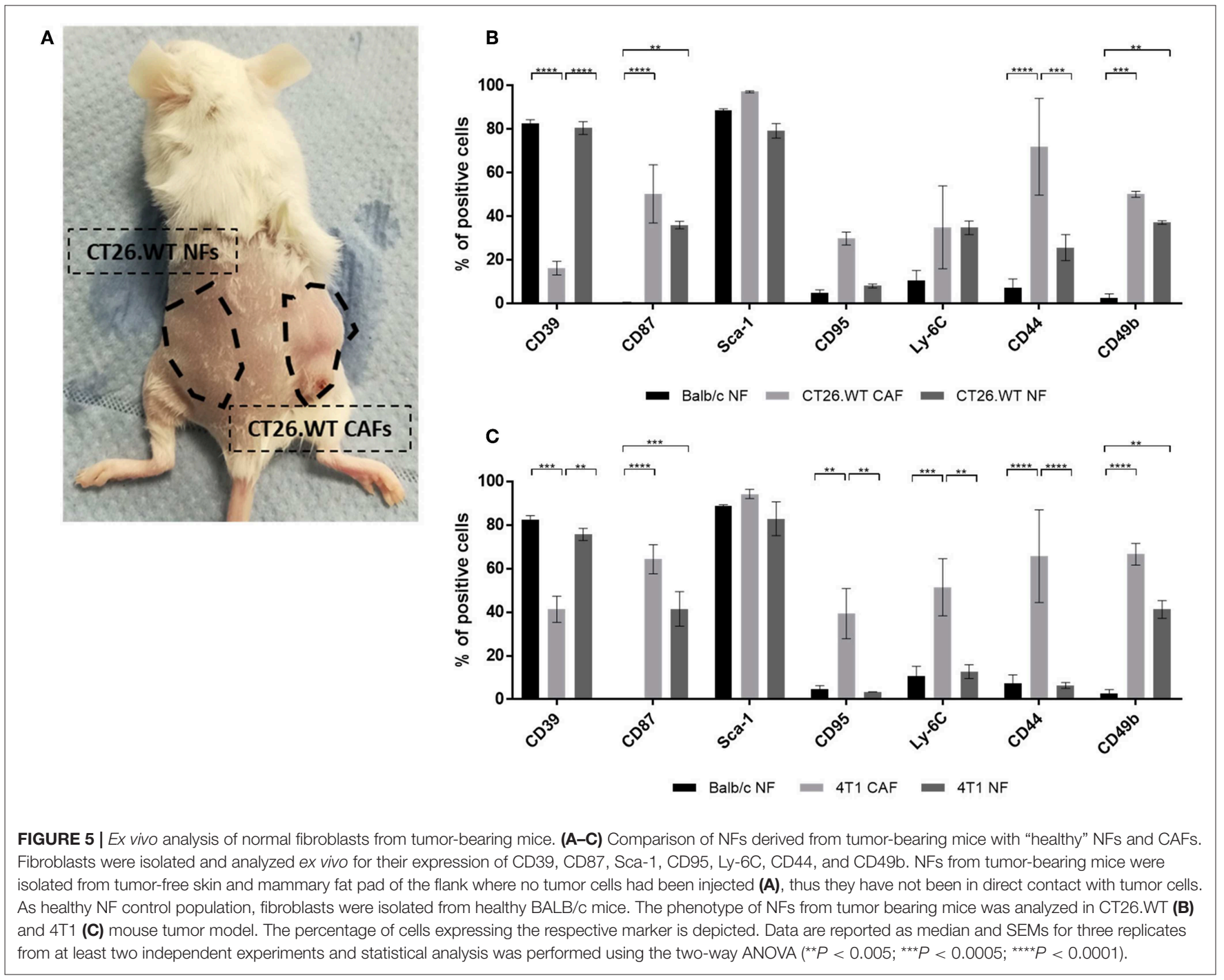

analyzed. Based on this screening, a panel of 62 markers was prepared and used for validation on isolated fibroblasts. Due to immunomagnetic sorting of the target cells, the data quality could be enhanced by removing non-fibroblasts, background noise, and unspecific signals. The markers EphA2, GARP, MHC class II, Rae-1a/b/g, CD80, CD87, CD106, CD123, and CD150 were detected to be expressed on 4T1- and CT26.WT-derived fibroblasts, whereas CD39 was detected specifically in normal fibroblasts. In addition, the expression patterns of CD95 and Ly-6C could be validated.
To the best of our knowledge, NF-associated markers that could facilitate identification of CAFs were lacking to date. We identified CD39 as the only cell surface marker specifically overexpressed in NFs based on these 233 candidates. CD39 is also known as ecto-nucleoside triphosphate diphosphohydrolase 1 (E-NTPDase1), and converts ATP into AMP when ATP has been released to the extracellular space $(30,31)$. It has been related to cancer mainly as tumor promoting molecule $(32,33)$ and is described to be expressed on immunomodulatory cells such as Tregs, functioning as immunosuppressive marker $(34,35)$. 
Moreover, its function is closely associated with the function of CD73 $(30,34)$. Yet, CD73 expression was not found to be correlated with CD39 in fibroblasts. Our findings suggested that CD39 is expressed at significantly lower levels in cancerassociated fibroblasts of $\mathrm{BALB} / \mathrm{c}$ mice as compared to normal "healthy" fibroblasts of BALB/c dermal and mammary fat pad tissues. This was surprising with respect to the described role of CD39 in cancer (32-34).

Next to the NF-marker CD39, our screenings revealed several markers found significantly overexpressed among CAFs or subpopulations thereof. CD87, showing the highest specificity for CAFs, is the receptor for urokinase-type plasminogen activator (uPA), and is part of the plasminogen activation system (36). The serine protease uPA is capable of degrading extracellular matrix proteins and is involved in tissue remodeling processes that occur e.g., in wound healing (37-40). Furthermore, the uPA/uPAR interaction has been described to be involved in cancer, where matrix remodeling and MMPs have been known as key players in cancer progression for years (41-43). Therefore, it nicely matches with the finding that CD87 was overrepresented in populations of CAFs in BALB/c mice but hardly expressed in normal fibroblasts. Interactions of uPA and UPAR have been described to be involved in the process of metastasizing $(37,43,44)$. It is possible that CD87 expression detected in NFs from tumor-bearing mice is associated to these observations and represents a premature sign of metastases.

Among the candidate markers correlating with the CAF phenotype, CD95 was identified in addition. CD95 is also known as death receptor Fas/APO-1 and has been associated with cancers in humans and mice for over a decade (45-50). Despite its contribution to the development of the immune system and regulation of immune responses, CD95 is reported to be involved in immune evasion of cancer cells and resistance to therapy (51-55). Interestingly, cancer cells apply different evasion mechanisms of CD95-induced apoptosis: reports showed that CD95 can be downregulated, but CD95 sensitivity can also be reduced by e.g., blocking of CD95 receptor and ligand binding (through soluble CD95L or soluble decoy receptor 3) or alteration of CD95 signaling pathway $(51,52,54,56-58)$. In this study, CD95 was found overrepresented in 4T1- and CT26.WT-CAFs ex vivo. Upon in vitro propagation, CD95 expression was unaffected in CAFs while NFs showed a significant upregulation of CD95 expression. Proliferation analysis of NFs showed a significant increase of CD95 expressing cells upon proliferation. It remains unclear whether CD95 signaling is altered in CAFs, comparable to the immune evasion mechanisms described in tumor cells. This could explain an inducibility in CD95 sensitive NFs but not in CD95-induced apoptosis-resistant CAFs.

Ly-6C is a characteristic molecule for myeloid cells $(59,60)$ and expressed on $\mathrm{T}$ cell subsets $(61,62)$, but was also found to be significantly overrepresented on cancer-associated fibroblasts ex vivo. The function of Ly-6C remains widely unknown, yet, it is mainly associated with the immune system, and inflammatory events (63-65). We identified Ly-6C as characteristic marker for CAFs, which is in line with current literature (66-68). Ly6C has been introduced as cell surface marker characteristic for a subpopulation of activated (cancer-associated) fibroblasts, termed inflammatory CAFs [iCAFs; (66-68)]. However, our data suggested Ly-6C to be a general fibroblast marker upregulated upon activation or proliferation and generally overexpressed in CAFs. This observation was only possible because in our study healthy NFs were always used as matched controls, rather than using putative progenitor cells $(67,68)$ or basing the observations on the expression of CAF-associated markers alone $(5,15-20)$.

Much like CD95 and Ly-6C, the glycoprotein CD44 was significantly overrepresented in CAFs isolated from $4 \mathrm{~T} 1$, and CT26.WT tumors. This marker has been related to different processes in cancer for decades $(66,69-71)$. Interestingly, this dramatically changed upon in vitro propagation and significant overrepresentation was found in NFs subsequently, yet, at markedly lower overall levels than observed for CAFs. Like Ly6C, CD44 expression was found upregulated in proliferating NFs while quiescent NFs did not show a CD44 expression in vitro. These data suggested a correlation of CD44 expression to proliferative activity, matching reports of CD44 expression in activated fibroblasts involved in inflammation and wound healing processes $(72,73)$. In contrast, CD44 levels in CAFs propagated in vitro were decreased, pointing at other functions of CD44 in CAFs. In a study of the association of CD44expressing CAFs and cancer cell stemness (5), it was shown that stromal CD44 expression was predominantly found in hypoxic areas of tumor tissue. Thus, if CD44 in CAFs is associated with or even induced by hypoxic conditions, it would explain that CD44 expression in CAFs propagated under normoxic in vitro conditions will downregulate CD44, despite actively proliferating. The authors of this study also tested this hypothesis and cultured CAFs under hypoxic conditions. Surprisingly, they saw even a decrease of CD44 expression of CAFs cultured under hypoxic conditions as compared to CAFs under normoxia. However, in their setting, CD44 expression was induced under hypoxic and hyponutritional conditions.

Interestingly, the widely used CAF-associated cell surface molecules PDGFR $\alpha$ and PDGFR $\beta$ (27-29) were not identified as CAF-specific candidates. We found PDGFR expression among fractions of NFs, yet, to a lower extent compared to CAFs (data not shown). This suggested that PDGFRs might not represent the best markers for identification and isolation of CAFs from solid tumors.

Most reports on CAF function for example use in vitro assays to study CAFs and their interaction with other cell types $(5,16,68,74,75)$. However, cultivation could potentially affect the behavior of cells as reflected by altered expression of characteristic markers among others. Notably, regulation of expression patterns could also have functional implications. According to our results depicted in Figure 3, upon cultivation CAFs widely preserved their specific expression patterns of markers identified before. On the contrary, NFs preserved expression of their characteristic marker CD39, but upregulated CAF-associated markers upon in vitro propagation. In light of the proliferation assay results, the CAF-associated markers are suggested to be surrogate markers for activation and proliferation among fibroblasts. Thus, those markers could be constantly expressed in CAFs which are thought to be 
highly activated fibroblasts and the expression is not affected in vitro, while expression of activation-associated markers is upregulated in NFs upon propagation and culture conditions that induce proliferation.

We found CD39 expression as being a characteristic feature of NFs, while expression of CAF-associated markers seemed to be also induced upon proliferation. Accordingly, we expected low expression of CAF-associated markers in NFs isolated from healthy tissues of tumor-bearing mice. Indeed, the markers CD44, CD95, and Ly-6C were expressed at low levels in NFs from tumor-bearing mice, suggesting a mainly activation- and proliferation-associated role and regulation. However, the markers CD49b, and CD87 were significantly upregulated in NFs from tumor-bearing mice. This demonstrated an independent, disease-associated role of those markers. Moreover, this showed that tumors can have a systemic effect on fibroblasts, even before they have metastasized. These findings could pave the way for identification of fibroblasts and markers in pre-metastatic lesions. For our study, we employed widely used cell line-induced tumor models. In future studies, these findings will have to be evaluated for general applicability using genetically or chemically induced tumor models.

Taken together, by employing a workflow optimized to immunomagnetically isolate fibroblasts from solid tissues and using appropriate healthy control cell populations, we have identified CD39 as novel cell surface marker for NFs as well as CD44, CD49b, CD87, CD95, and Ly$6 \mathrm{C}$ as CAF-associated markers. Our data suggested an association of the CAF markers, most strikingly CD87 and Ly-6C, with fibroblast activation and proliferation. Furthermore, the widely known stem cell-associated marker Sca-1 and the expression level of CD90.2 correlated with fibroblast proliferation in our assays. We demonstrated that induction of CD49b and CD87 expression is triggered in NFs

\section{REFERENCES}

1. Kalluri R, Zeisberg M. Fibroblasts in cancer. Nat Rev Cancer. (2006) 6:392401. doi: $10.1038 / \mathrm{nrc1} 877$

2. Orimo A, Weinberg RA. Heterogeneity of stromal fibroblasts in tumors. Cancer Biol Ther. (2007) 6:618-9. doi: 10.4161/cbt.6.4.4255

3. Hanahan D, Weinberg RA. Hallmarks of cancer: the next generation. Cell. (2011) 144:646-74. doi: 10.1016/j.cell.2011.02.013

4. Pietras K, Ostman A. Hallmarks of cancer: interactions with the tumor stroma. Exp Cell Res. (2010) 316:1324-31. doi: 10.1016/j.yexcr.2010.02.045

5. Kinugasa Y, Matsui T, Takakura N. CD44 expressed on cancer-associated fibroblasts is a functional molecule supporting the stemness and drug resistance of malignant cancer cells in the tumor microenvironment. Stem Cells. (2014) 32:145-56. doi: 10.1002/stem.1556

6. Lee KW, Yeo SY, Sung CO, Kim SH. Twist1 is a key regulator of cancer-associated fibroblasts. Cancer Res. (2015) 75:7385. doi: 10.1158/0008-5472.CAN-14-0350

7. Taddei ML, Cavallini L, Ramazzotti M, Comito G, Pietrovito L, Morandi A, et al. Stromal-induced downregulation of miR-1247 promotes prostate cancer malignancy. J Cell Physiol. (2019) 234:8274-85. doi: 10.1002/jcp.27679

8. Cirri P, Chiarugi P. Cancer-associated-fibroblasts and tumour cells: a diabolic liaison driving cancer progression. Cancer Meta Rev. (2012) 31:195208. doi: 10.1007/s10555-011-9340-x from healthy tissues by systemic cancer induced effects in tumor-bearing mice.

\section{DATA AVAILABILITY}

All datasets generated for this study are included in the manuscript and/or the Supplementary Files.

\section{ETHICS STATEMENT}

This study was carried out in accordance with the recommendations of European and German guidelines for the care of laboratory animals. The protocol was approved by the ethical committee on animal care and use in North Rhine-Westphalia (approval number: 84_02.04.2014.A325).

\section{AUTHOR CONTRIBUTIONS}

$\mathrm{DA}, \mathrm{AB}, \mathrm{SW}$, and $\mathrm{OH}$ conceived and designed the experiments. $\mathrm{DA}, \mathrm{AL}$, and $\mathrm{UH}$ performed the experiments. DA, AL, and $\mathrm{OH}$ analyzed the data. $\mathrm{DA}, \mathrm{AB}$, and $\mathrm{OH}$ wrote the manuscript.

\section{ACKNOWLEDGMENTS}

We are grateful to Lena Willnow for excellent technical assistance. Furthermore, we thank Merlin Siewert and Frank Single for their support on animal care and work. In addition, we thank Andrea Thiele and the MMLS master program at HAN University of Applied Sciences for their support.

\section{SUPPLEMENTARY MATERIAL}

The Supplementary Material for this article can be found online at: https://www.frontiersin.org/articles/10.3389/fonc. 2019.00716/full\#supplementary-material
9. Morandi A, Giannoni E, Chiarugi P. Nutrient exploitation within the tumor-stroma metabolic crosstalk. Trends Cancer. (2016) 2:736-46. doi: 10.1016/j.trecan.2016.11.001

10. Ippolito L, Morandi A, Taddei ML, Parri M, Comito G, Iscaro A, et al. Cancer-associated fibroblasts promote prostate cancer malignancy via metabolic rewiring and mitochondrial transfer. Oncogene. (2019) 38:533955. doi: 10.1038/s41388-019-0805-7

11. Shimoda M, Mellody KT, Orimo A. Carcinoma-associated fibroblasts are a rate-limiting determinant for tumour progression. Sem Cell Develop Biol. (2010) 21:19-25. doi: 10.1016/j.semcdb.2009. 10.002

12. Mueller MM, Fusenig NE. Friends or foes - bipolar effects of the tumour stroma in cancer. Nat Rev Cancer. (2004) 4:839-49. doi: 10.1038/nrc1477

13. Ostman A, Augsten M. Cancer-associated fibroblasts and tumor growthbystanders turning into key players. Curr Opin Genet Develop. (2009) 19:6773. doi: $10.1016 /$ j.gde.2009.01.003

14. Sugimoto H, Mundel TM, Kieran MW, Kalluri R. Identification of fibroblast heterogeneity in the tumor microenvironment. Cancer Biol Ther. (2006) 5:1640-6. doi: 10.4161/cbt.5.12.3354

15. Costa A, Kieffer Y, Scholer-Dahirel A, Pelon F, Bourachot B, Cardon M, et al. Fibroblast heterogeneity and immunosuppressive environment in human breast cancer. Cancer Cell. (2018) 33:463-79.e10. doi: 10.1016/j.ccell.2018.01.011 
16. Herrera M, Islam AB, Herrera A, Martin P, Garcia V, Silva J, et al. Functional heterogeneity of cancer-associated fibroblasts from human colon tumors shows specific prognostic gene expression signature. Clin Cancer Res. (2013) 19:5914-26. doi: 10.1158/1078-0432.CCR-13-0694

17. Kong J, Zhao H, Shang Q, Ma Z, Kang N, Tan J, et al. Establishment and characterization of a carcinoma-associated fibroblast cell line derived from a human salivary gland adenoid cystic carcinoma. Cell Commun Adhes. (2018) 24:11-8. doi: 10.1080/15419061.2018.1464000

18. Majety M, Pradel LP, Gies M, Ries CH. Fibroblasts influence survival and therapeutic response in a 3D co-culture model. PloS ONE. (2015) 10:e0127948. doi: 10.1371/journal.pone.0127948

19. Nazareth MR, Broderick L, Simpson-Abelson MR, Kelleher RJ Jr, Yokota SJ, Bankert RB. Characterization of human lung tumor-associated fibroblasts and their ability to modulate the activation of tumor-associated T cells. J Immunol. (2007) 178:5552-62. doi: 10.4049/jimmunol.178.9.5552

20. Zhang A, Qian Y, Ye Z, Chen H, Xie H, Zhou L, et al. Cancer-associated fibroblasts promote M2 polarization of macrophages in pancreatic ductal adenocarcinoma. Cancer Med. (2017) 6:463-70. doi: 10.1002/cam4.993

21. Arndt R, Stark R, Thiele HG. Detection and molecular characterization of the thymus-brain antigen in human brain. Immunology. (1977) 33:101-7.

22. Cotmore SF, Crowhurst SA, Waterfield MD. Purification of Thy-1-related glycoproteins from human brain and fibroblasts: comparisons between these molecules and murine glycoproteins carrying Thy-1.1 and Thy-1.2 antigens. Euro J Immunol. (1981) 11:597-603. doi: 10.1002/eji.1830110802

23. Pont S. Thy-1: a lymphoid cell subset marker capable of delivering an activation signal to mouse T lymphocytes. Biochimie. (1987) 69:31520. doi: 10.1016/0300-9084(87)90022-8

24. Liu Y, Cao X. Characteristics and significance of the pre-metastatic niche. Cancer Cell. (2016) 30:668-81. doi: 10.1016/j.ccell.2016.09.011

25. Psaila B, Lyden D. The metastatic niche: adapting the foreign soil. Nat Rev Cancer. (2009) 9:285-93. doi: 10.1038/nrc2621

26. Sappino AP, Skalli O, Jackson B, Schurch W, Gabbiani G. Smooth-muscle differentiation in stromal cells of malignant and non-malignant breast tissues. Int J Cancer. (1988) 41:707-12. doi: 10.1002/ijc.2910410512

27. Sasaki K, Sugai T, Ishida K, Osakabe M, Amano H, Kimura H, et al. Analysis of cancer-associated fibroblasts and the epithelial-mesenchymal transition in cutaneous basal cell carcinoma, squamous cell carcinoma, and malignant melanoma. Hum Pathol. (2018) 79:1-8. doi: 10.1016/j.humpath.2018.03.006

28. Micke P, Ostman A. Tumour-stroma interaction: cancer-associated fibroblasts as novel targets in anti-cancer therapy? Lung Cancer. (2004) 45:S16375. doi: 10.1016/j.lungcan.2004.07.977

29. Pietras K, Sjoblom T, Rubin K, Heldin CH, Ostman A. PDGF receptors as cancer drug targets. Cancer Cell. (2003) 3:43943. doi: 10.1016/S1535-6108(03)00089-8

30. Antonioli L, Pacher P, Vizi ES, Hasko G. CD39 and CD73 in immunity and inflammation. Trends Mol Med. (2013) 19:35567. doi: 10.1016/j.molmed.2013.03.005

31. Heine P, Braun N, Sevigny J, Robson SC, Servos J, Zimmermann H. The Cterminal cysteine-rich region dictates specific catalytic properties in chimeras of the ectonucleotidases NTPDase1 and NTPDase2. Euro J Biochem. (2001) 268:364-73. doi: 10.1046/j.1432-1327.2001.01896.x

32. Feng L, Sun X, Csizmadia E, Han L, Bian S, Murakami T, et al. Vascular CD39/ENTPD1 directly promotes tumor cell growth by scavenging extracellular adenosine triphosphate. Neoplasia. (2011) 13:206-16. doi: 10.1593/neo.101332

33. Kunzli BM, Bernlochner MI, Rath S, Kaser S, Csizmadia E, Enjyoji $\mathrm{K}$, et al. Impact of CD39 and purinergic signalling on the growth and metastasis of colorectal cancer. Purinergic Signal. (2011) 7:23141. doi: 10.1007/s11302-011-9228-9

34. Allard B, Longhi MS, Robson SC, Stagg J. The ectonucleotidases CD39 and CD73: novel checkpoint inhibitor targets. Immunol Rev. (2017) 276:12144. doi: 10.1111/imr.12528

35. Borsellino G, Kleinewietfeld M, Di Mitri D, Sternjak A, Diamantini A, Giometto R, et al. Expression of ectonucleotidase CD39 by Foxp3+ Treg cells: hydrolysis of extracellular ATP and immune suppression. Blood. (2007) 110:1225-32. doi: 10.1182/blood-2006-12-064527

36. Vassalli JD, Sappino AP, Belin D. The plasminogen activator/plasmin system. J Clin Investig. (1991) 88:1067-72. doi: 10.1172/JCI115405
37. Mahmood N, Mihalcioiu C, Rabbani SA. Multifaceted role of the urokinase-type plasminogen activator (uPA) and its receptor (uPAR): diagnostic, prognostic, and therapeutic applications. Front Oncol. (2018) 8:24. doi: $10.3389 /$ fonc. 2018.00024

38. Noh H, Hong S, Huang S. Role of urokinase receptor in tumor progression and development. Theranostics. (2013) 3:487-95. doi: 10.7150/thno.4218

39. Schafer BM, Maier K, Eickhoff U, Todd RF, Kramer MD. Plasminogen activation in healing human wounds. Am J Pathol. (1994) 144:1269-80.

40. Sulniute R, Shen Y, Guo YZ, Fallah M, Ahlskog N, Ny L, et al. Plasminogen is a critical regulator of cutaneous wound healing. Thromb Haemost. (2016) 115:1001-9. doi: 10.1160/TH15-08-0653

41. Estreicher A, Muhlhauser J, Carpentier JL, Orci L, Vassalli JD. The receptor for urokinase type plasminogen activator polarizes expression of the protease to the leading edge of migrating monocytes and promotes degradation of enzyme inhibitor complexes. J Cell Biol. (1990) 111:783-92. doi: 10.1083/jcb.111.2.783

42. Jo M, Takimoto S, Montel V, Gonias SL. The urokinase receptor promotes cancer metastasis independently of urokinase-type plasminogen activator in mice. Am J Pathol. (2009) 175:190-200. doi: 10.2353/ajpath.2009.081053

43. Schuler PJ, Bendszus M, Kuehnel S, Wagner S, Hoffmann TK, Goldbrunner $\mathrm{R}$, et al. Urokinase plasminogen activator, uPAR, MMP-2, and MMP-9 in the C6-glioblastoma rat model. In vivo. (2012) 26:571-6.

44. Andreasen PA, Kjoller L, Christensen L, Duffy MJ. The urokinase-type plasminogen activator system in cancer metastasis: a review. Int $J$ Cancer. (1997) 72:122. doi: 10.1002/(SICI)1097-0215(19970703)72:1<1::AID-IJC1>3.0.CO;2-Z

45. Barnhart BC, Legembre P, Pietras E, Bubici C, Franzoso G, Peter ME. CD95 ligand induces motility and invasiveness of apoptosis-resistant tumor cells. EMBO J. (2004) 23:3175-85. doi: 10.1038/sj.emboj.7600325

46. Chen L, Park SM, Tumanov AV, Hau A, Sawada K, Feig C, et al. CD95 promotes tumour growth. Nature. (2010) 465:4926. doi: $10.1038 /$ nature 09075

47. Debatin KM, Krammer PH. Death receptors in chemotherapy and cancer. Oncogene. (2004) 23:2950-66. doi: 10.1038/sj.onc.1207558

48. Nagata S. Fas ligand-induced apoptosis. Ann Rev Genet. (1999) 33:2955. doi: 10.1146/annurev.genet.33.1.29

49. Peshes-Yaloz N, Rosen D, Sondel PM, Krammer PH, Berke G. Up-regulation of Fas (CD95) expression in tumour cells in vivo. Immunology. (2007) 120:502-11. doi: 10.1111/j.1365-2567.2006.02521.x

50. Peter ME, Legembre P, Barnhart BC. Does CD95 have tumor promoting activities? Biochim Biophys Acta. (2005) 1755:2536. doi: 10.1016/j.bbcan.2005.01.001

51. Ametller E, Garcia-Recio S, Costamagna D, Mayordomo C, FernandezNogueira P, Carbo N, et al. Tumor promoting effects of CD95 signaling in chemoresistant cells. Mol Cancer. (2010) 9:161. doi: 10.1186/1476-4598-9-161

52. Buhling F, Wille A, Rocken C, Wiesner O, Baier A, Meinecke I, et al. Altered expression of membrane-bound and soluble CD95/Fas contributes to the resistance of fibrotic lung fibroblasts to FasL induced apoptosis. Respir Res. (2005) 6:37. doi: 10.1186/1465-9921-6-37

53. Debatin KM. The role of CD95 system in chemotherapy. Drug Resist Updat. (1999) 2:85-90. doi: 10.1054/drup.1999.0073

54. Friesen C, Fulda S, Debatin KM. Deficient activation of the CD95 (APO-1/Fas) system in drug-resistant cells. Leukemia. (1997) 11:183341. doi: 10.1038/sj.leu.2400827

55. Peter ME, Hadji A, Murmann AE, Brockway S, Putzbach W, Pattanayak A, et al. The role of CD95 and CD95 ligand in cancer. Cell Death Differ. (2015) 22:549-59. doi: 10.1038/cdd.2015.3

56. Igney FH, Krammer PH. Death and anti-death: tumour resistance to apoptosis. Nat Rev Cancer. (2002) 2:277-88. doi: 10.1038/nrc776

57. Pitti RM, Marsters SA, Lawrence DA, Roy M, Kischkel FC, Dowd P, et al. Genomic amplification of a decoy receptor for Fas ligand in lung and colon cancer. Nature. (1998) 396:699-703. doi: 10.1038/25387

58. Strand S, Vollmer P, van den Abeelen L, Gottfried D, Alla V, Heid H, et al. Cleavage of CD95 by matrix metalloproteinase-7 induces apoptosis resistance in tumour cells. Oncogene. (2004) 23:3732-6. doi: 10.1038/sj.onc.1207387

59. Jutila MA, Kroese FG, Jutila KL, Stall AM, Fiering S, Herzenberg LA, et al. Ly-6C is a monocyte/macrophage and endothelial cell differentiation antigen regulated by interferon-gamma. Euro J Immunol. (1988) 18:181926. doi: $10.1002 /$ eji.1830181125 
60. Takikawa O, Oku T, Ito N, Ushio $\mathrm{Y}$, Yamamoto $\mathrm{N}$, Yoneda $\mathrm{Y}$, et al. Multiple expression of Ly-6C and accumulation of a Ly-6C pre-mRNA in activated macrophages involved in rejection of an allografted tumor. Biochem Biophys Res Commun. (1996) 226:247-53. doi: 10.1006/bbrc. 1996.1341

61. McHeyzer-Williams LJ, McHeyzer-Williams MG. Developmentally distinct Th cells control plasma cell production in vivo. Immunity. (2004) 20:23142. doi: 10.1016/S1074-7613(04)00028-7

62. Pihlgren M, Dubois PM, Tomkowiak M, Sjogren T, Marvel J. Resting memory $\mathrm{CD} 8+\mathrm{T}$ cells are hyperreactive to antigenic challenge in vitro. J Exp Med. (1996) 184:2141-51. doi: 10.1084/jem.184.6.2141

63. Schlueter AJ, Krieg AM, De Vries P, Li X. B cells express Ly-6C in a Th1 but not Th2 cytokine environment. J Interferon Cytokine Res. (2002) 22:799806. doi: $10.1089 / 107999002320271396$

64. Yang J, Zhang L, Yu C, Yang XF, Wang H. Monocyte and macrophage differentiation: circulation inflammatory monocyte as biomarker for inflammatory diseases. Biomarker Res. (2014) 2:1. doi: 10.1186/20507771-2-1

65. Zigmond E, Varol C, Farache J, Elmaliah E, Satpathy AT, Friedlander G, et al. Ly6C hi monocytes in the inflamed colon give rise to proinflammatory effector cells and migratory antigen-presenting cells. Immunity. (2012) 37:107690. doi: 10.1016/j.immuni.2012.08.026

66. Bernard V, Semaan A, Huang J, San Lucas FA, Mulu FC, Stephens BM, et al. Single-cell transcriptomics of pancreatic cancer precursors demonstrates epithelial and microenvironmental heterogeneity as an early event in neoplastic progression. Clin Cancer Res. (2018). doi: 10.1101/306134

67. Biffi G, Oni TE, Spielman B, Hao Y, Elyada E, Park Y, et al. IL1Induced JAK/STAT signaling is antagonized by TGFbeta to shape CAF heterogeneity in pancreatic ductal adenocarcinoma. Cancer Discov. (2019) 9:282-301. doi: 10.1158/2159-8290.CD-18-0710

68. Ohlund D, Handly-Santana A, Biffi G, Elyada E, Almeida AS, Ponz-Sarvise $\mathrm{M}$, et al. Distinct populations of inflammatory fibroblasts and myofibroblasts in pancreatic cancer. J Exp Med. (2017) 214:579-96. doi: 10.1084/jem. 20162024
69. Kaufmann M, Heider KH, Sinn HP, von Minckwitz G, Ponta H, Herrlich P. CD44 variant exon epitopes in primary breast cancer and length of survival. Lancet. (1995) 345:615-9. doi: 10.1016/S0140-6736(95)90521-9

70. Mulder JW, Kruyt PM, Sewnath M, Oosting J, Seldenrijk CA, Weidema WF, et al. Colorectal cancer prognosis and expression of exon-v6-containing CD44 proteins. Lancet. (1994) 344:1470-2. doi: 10.1016/S0140-6736(94)90290-9

71. Rall CJ, Rustgi AK. CD44 isoform expression in primary and metastatic pancreatic adenocarcinoma. Cancer Res. (1995) 55:1831-5.

72. Mikecz K, Brennan FR, Kim JH, Glant TT. Anti-CD44 treatment abrogates tissue oedema and leukocyte infiltration in murine arthritis. Nat Med. (1995) 1:558-63. doi: 10.1038/nm0695-558

73. Misra S, Hascall VC, Markwald RR, Ghatak S. Interactions between hyaluronan and its receptors (CD44, RHAMM) regulate the activities of inflammation and cancer. Front Immunol. (2015) 6:201. doi: 10.3389/fimmu.2015.00201

74. Dorsam B, Bosl T, Reiners KS, Barnert S, Schubert R, Shatnyeva $\mathrm{O}$, et al. Hodgkin lymphoma-derived extracellular vesicles change the secretome of fibroblasts toward a CAF phenotype. Front Immunol. (2018) 9:1358. doi: 10.3389/fimmu.2018.01358

75. Kuen J, Darowski D, Kluge T, Majety M. Pancreatic cancer cell/fibroblast coculture induces M2 like macrophages that influence therapeutic response in a 3D model. PloS ONE. (2017) 12:e0182039. doi: 10.1371/journal.pone.0182039

Conflict of Interest Statement: DA, AL, UH, SW, AB, and $\mathrm{OH}$ are or were full time employees of Miltenyi Biotec GmbH.

Copyright $\odot 2019$ Agorku, Langhammer, Heider, Wild, Bosio and Hardt. This is an open-access article distributed under the terms of the Creative Commons Attribution License (CC BY). The use, distribution or reproduction in other forums is permitted, provided the original author(s) and the copyright owner(s) are credited and that the original publication in this journal is cited, in accordance with accepted academic practice. No use, distribution or reproduction is permitted which does not comply with these terms. 